\title{
Single and Multiple variables control using Tree Physiology Optimization
}

\author{
A.Hanif Halim*, and $I$. Ismail \\ Electrical and Electronic Engineering Department, Universiti Teknologi PETRONAS, \\ 31750 Tronoh, Perak, Malaysia
}

\begin{abstract}
This paper presents the tuning of single-input single-output (SISO), and multiple-input multiple-output (MIMO) control system using Tree Physiology Optimization (TPO). TPO is a metaheuristic optimization algorithm that has a clustered diversification search strategy inspired from plant shoots growth. The clustered diversification is referred as tree branch and leaves. The exploration is amplified from roots growth counterparts. In the proposed method, each shoot from each branch search for possible solution in parallel and the fitness is evaluated based on all best values found by branch search. The proposed algorithm is also compared with deterministic gradient-free algorithm: Nelder-Mead simplex (NMS) and another metaheuristic algorithm: Particle Swarm Optimization (PSO). Results shown that TPO is able to find good PID parameters with lesser settling time for SISO and MIMO process. NMS is also able to find good PID parameters for SISO with lesser performance index, however not able to find better solution in MIMO control. PSO converged prematurely in SISO control and has high overshoot for MIMO control optimization.
\end{abstract}

\section{Introduction}

To date, the tuning of PID controller is still in extensive research. There are many PID controllers in practice that are not tuned properly [1]. Such tuning is normally carried out with trial and error method which is time consuming [2]. In addition, the multivariable process that involved controlling of many input and output variables are regularly practiced in industrial plant [2]. Therefore such high complexities of process need to be properly controlled. The multiple input-output control systems are also defined as MIMO control system. In contrast, the single input-output control system is denoted as SISO control. SISO PID control is available in most commercial controllers [3] and cited in many literatures as described in [4]. There are many tuning method proposed in the literatures. Among the most implemented method is metaheuristic algorithm. Metaheuristic algorithm is proven to be more efficient in computation time and performance [7].

*Corresponding author: abdul_g01772@utp.edu.my 


\section{Optimization algorithm}

There are essentially two types of optimization algorithms: deterministic and stochastic [8]. Deterministic algorithm has a repeatable path to find a solution by a given input. Deterministic algorithm is further divided into two approaches: gradient-based and gradient-free search method. Gradient-based algorithm uses gradient information to search for optimal solution, which may trapped into local optimum especially in multimodal problem. In contrast, gradient-free algorithm eliminate this disadvantage and able to search towards global optimum. Simplex algorithm is one example of gradient-free algorithm [10] and the algorithm is proven to be more effective than GA and Simulated Annealing (SA) [9]. Another section of optimization algorithm is the stochastic algorithm, which is also denoted as heuristic or metaheuristic algorithm. Metaheuristic algorithm has unique properties of search that incorporate two components: diversification and intensification [8]. Diversification is to generate a diverse solution in order to explore the search space. Intensification is to focus the search space in local region by exploiting the information of current good solution of respective region. Some examples of metaheuristic algorithms are GA, SA, PSO, FFA, and TPO. In this paper, three optimization algorithms for solving SISO and MIMO control system will be discussed. The algorithms are Nelder-Mead Simplex algorithm (NMS) [11], Particle Swarm Optimization (PSO) [12], and Tree Physiology Optimization (TPO) [16]. The characteristic of each algorithm is summarized in Table 1.

Table 1: Overview of proposed algorithm

\begin{tabular}{|l|c|l|}
\hline Num & Algo. (year) & \multicolumn{1}{c|}{ Main features } \\
\hline 1 & NMS (1965) & $\begin{array}{l}\text { Triangular Search driven with 3 main steps: } \\
\text { reflection, contraction and expansion [11,12] }\end{array}$ \\
\hline 2 & PSO (1995) & $\begin{array}{l}\text { Swarm-based algorithm. Solution is attracted to } \\
\text { local and global best in each iteration [9, 13]. }\end{array}$ \\
\hline 3 & TPO (2013) & $\begin{array}{l}\text { Plant growth inspired algorithm with shoots as } \\
\text { search agent. Shoots consist of defined leaves } \\
\text { and branches for parallel exploration. The } \\
\text { search is amplified by root-shoot correlation [16 } \\
-18] .\end{array}$ \\
\hline
\end{tabular}

The performance criterion for fitness evaluation of each algorithm is based on integral of absolute error (IAE) with response constraint as shown in (1).

$$
J=\int|e| d t+\int_{0}^{t_{\max }}\left(f_{u p}-x_{i}\right)+\int_{t_{1}}^{t_{\max }}\left(x_{i}-f_{\text {low }}\right)
$$

Whereas $f_{\text {up }}$ and $f_{\text {low }}$ are continuous function that define the upper and lower border of shaded region as in Fig. 1.

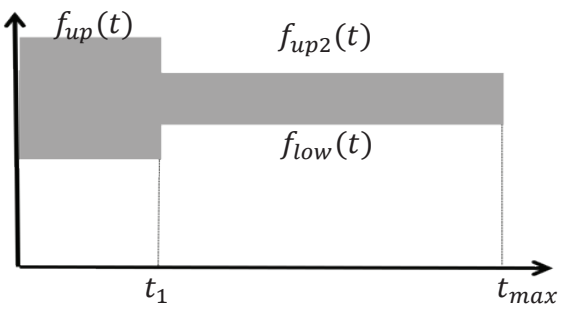

(a)

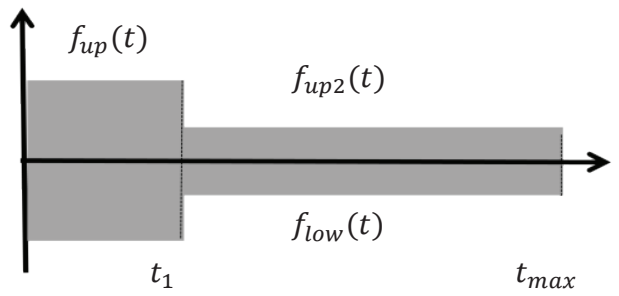

(b)

Fig. 1: Desired region for plant response with (a) step of active control loop and (b) passive control loop 
The constraint $f_{u p}$ consists of two borders $\left(f_{u p}\right.$ and $\left.f_{u p 2}\right) . x_{i}$ is the plant response at each iteration. Based on the figure, the settings of $f_{u p}(t), f_{l o w}(t), t_{1}$ and $t_{\text {max }}$ are specified by user. The performance criteria of tuned control parameters are based on response constraint and IAE, since the response constraint may assist the estimation to desired response region. In this paper, the response setting is set with $f_{\text {up }}=1.55 ; f_{\text {up } 2}=1.20 ; f_{\text {low }}=0.8 ; t_{1}=$ $1.6 ; t_{\max }=20$

\section{Application on SISO optimization}

The algorithm performance is compared with SISO of DC servo motor for position control as presented in [13] with transfer function defined in (2). The block diagram of closed loop PID control with online optimization is shown as in Fig. 2. The limitation of each controller parameter is set to $[0,5]$.

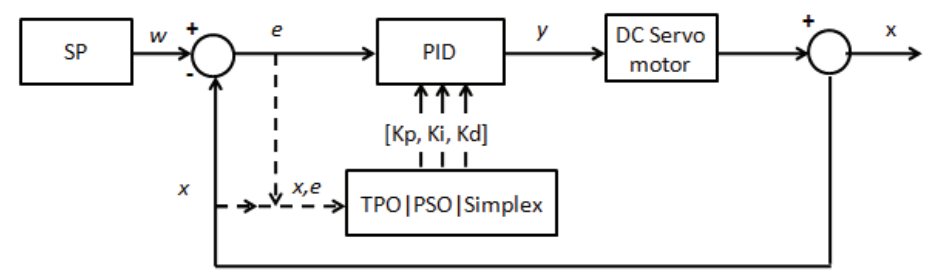

Fig. 2: Block diagram of closed loop controller tuning using proposed algorithm

$$
G_{\text {DC Servo Motor }}(s)=\frac{0.1}{0.01 s^{3}+0.09 s^{2}+0.21 s}
$$

Based on Fig. 2, the PID parameters are updated by proposed the algorithm in each iteration. The control performance is measured based on plant output $(x)$, that used to evaluate the constraint area and error value $(e)$ that determine the IAE criteria.

\subsection{Simulation result}

Each algorithm is set according to their original setting. The number of iteration for PSO and NMS is set to 30. For TPO, the number of branches and shoots leaves is set to 10 and 20 respectively. The step response of each algorithm is depicted in Table 2 and Fig. 3. The best value is highlighted with bold. It is observed that NMS has the lowest $J$ value (IAE with response constraint). All algorithms show merely the same rise time. TPO has the shortest settling time and converge in less than $\pm 1 \%$ of set value as shown in Figure $3 \mathrm{~b}$. PSO has the lowest overshoot, however with longer time characteristics

Table 2: Optimum PID based on each method

\begin{tabular}{llllllll}
\hline Method & $\mathrm{Kp}$ & $\mathrm{Ki}$ & $\mathrm{Kd}$ & $\begin{array}{l}\text { Rise } \\
\text { time }\end{array}$ & $\begin{array}{l}\text { Settling } \\
\text { time }\end{array}$ & $\begin{array}{l}\text { Overshoot } \\
\%\end{array}$ & $J$ \\
\hline NMS & 4.701 & 0.0384 & 0.0301 & $\mathbf{1 . 2 0}$ & 5.00 & 23 & $\mathbf{1 . 0 9}$ \\
\hline PSO & 3.577 & 0.158 & 0.005 & 1.22 & 7.60 & $\mathbf{1 6}$ & 1.27 \\
\hline TPO & 3.156 & 0.001 & 0.001 & 1.21 & $\mathbf{3 . 8 0}$ & 20 & 1.21 \\
\hline
\end{tabular}


(a)

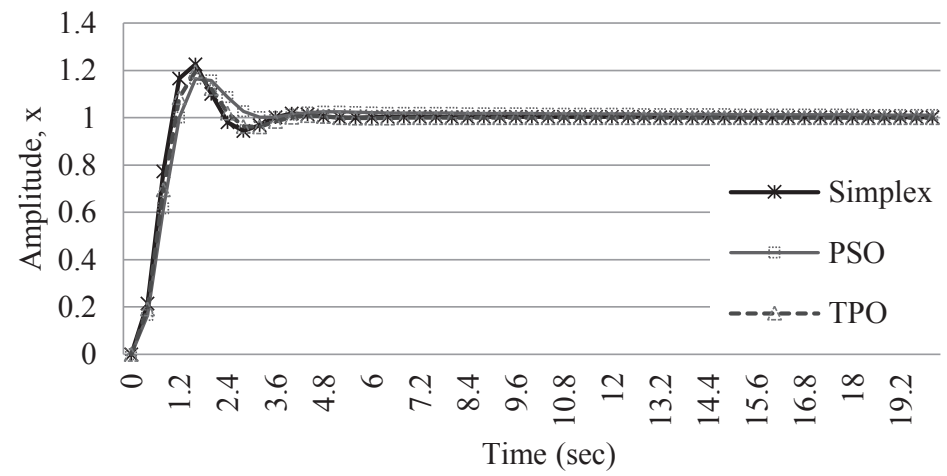

(b)

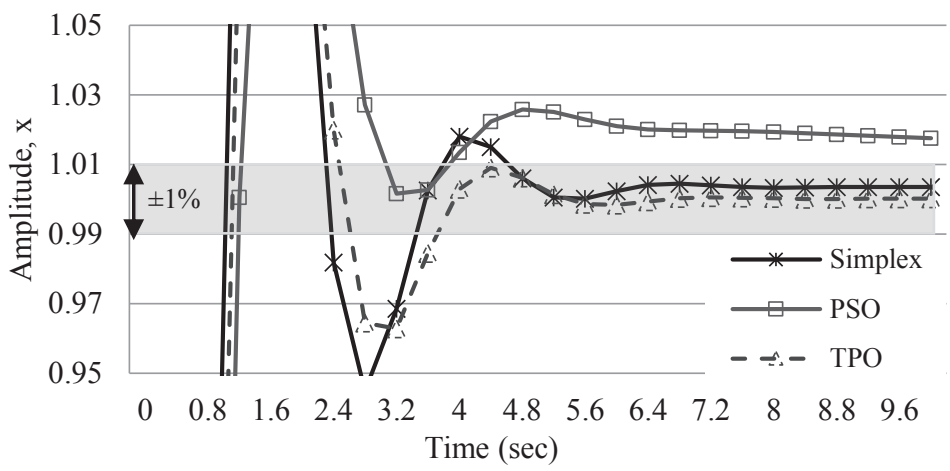

Fig. 3: (a) Optimized step response of each algorithm,

(b) Response of each method within $\pm 1 \%$ tolerance

\section{Application on MIMO optimization}

For multiple variable processes, the performance of each algorithm is compared with Wood and Berry distillation column control [14]. The control strategy for multivariable system is presented by Wood and Berry, which is to reduce the interaction between the control of the overhead and bottom products [14]. The input-output variable for MIMO system is expressed with output: $Y(s)=\left[\begin{array}{l}Y_{1}(s) \\ Y_{2}(s)\end{array}\right]$ and input: $U(s)=\left[\begin{array}{l}U_{1}(s) \\ U_{2}(s)\end{array}\right]$. The inputs of this process are the reflux and steam flow rates with outputs of overhead and bottoms composition.

$$
G(s)=\left[\begin{array}{ll}
G_{11}(s) & G_{12}(s) \\
G_{21}(s) & G_{22}(s)
\end{array}\right]=\left[\begin{array}{cc}
\frac{12.8 e^{-s}}{1+16.7 s} & \frac{-18.9 e^{-3 s}}{1+21 s} \\
\frac{6.6 e^{-7 s}}{1+10.9 s} & \frac{-19.4 e^{-3 s}}{1+14.4 s}
\end{array}\right]
$$

Two PID controllers with $i^{\text {th }}$ vector representation are designed for this problem as shown in (4). The vector is updated in each number of iteration for each proposed optimization algorithm. 


$$
C_{1,2 i}=\left[K_{P 1_{i}}, K_{I 1_{i}}, K_{D 1_{i}}, K_{P 2_{i}}, K_{I 2_{i}}, K_{D 2_{i}}\right]
$$

Whereas $C_{1,2 i}$ consists of $C_{1}$ that represents PID of $K_{P 1}, K_{I 1}, K_{D 1}$ and $C_{2}$ for $K_{P 2}, K_{I 2}, K_{D 2}$. Figure $4 \mathrm{~b}$ shows the MIMO control structure designed for this work.

(a)

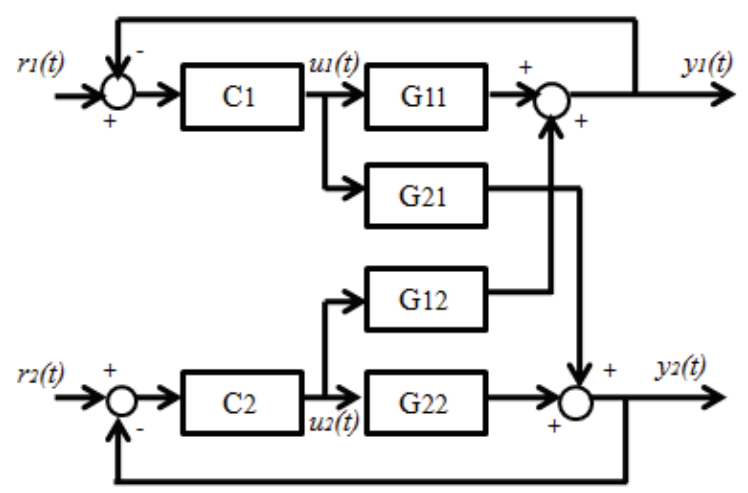

(b)

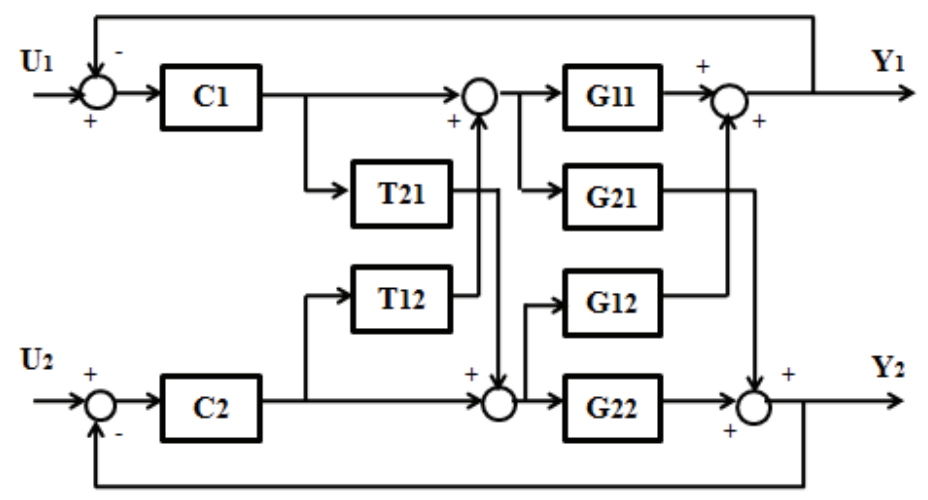

Fig. 4: (a) Block diagram of decentralized control of MIMO system,

(b) MIMO system with decoupler

In this paper, a simplified decoupling method is implemented. The best response is obtained by tuning of decoupler and PID controllers. A decoupling matrix, $T$ is evaluated with transfer matrix. The controller parameters are tuned based on predefined elements of T. The elements of simplified decoupling are $T_{11}=T_{22}=1$ and off-diagonal with, $T_{12}=$ $-\frac{G_{12}}{G_{11}}$ and $T_{21}=-\frac{G_{21}}{G_{22}}$. The range of each controller parameter is set according to min-max value Kp: $[-1.5,1.5], \mathrm{Ki}:[-0.15,0.15]$ and $\mathrm{Kd}:[-0.2,0.2]$ and constraint setting with $f_{u p}=$ $1.55 ; f_{\text {up } 2}=1.02 ; f_{\text {low }}=0.8 ; t_{1}=20 ; t_{\text {max }}=100$

\subsection{Simulation result}

The setting of each algorithm is set similar to SISO problem. The parameters of PID controller obtained by each algorithm and control loop are depicted in Table 2. The step response of each algorithm is shown in Table 3 and Fig.5. 
Table 2: Optimum PID based on each method for MIMO control

\begin{tabular}{lllllll}
\hline Method & $K_{P 1}$ & $K_{I 1}$ & $K_{D 1}$ & $K_{P 2}$ & $K_{I 2}$ & $K_{D 3}$ \\
\hline NMS & 0.3010 & 0.0633 & 0.0143 & -0.0383 & -0.0278 & -0.0502 \\
\hline PSO & 0.3052 & 0.0230 & 0.0040 & -0.1324 & -0.0394 & -0.0361 \\
\hline TPO & 0.3266 & 0.0413 & 0.3662 & -0.1277 & -0.0140 & -0.0733 \\
\hline
\end{tabular}

Table 3: Response properties of each algorithm

\begin{tabular}{|l|llll|llll|}
\hline & \multicolumn{5}{|c|}{ Step $y_{11}$} & \multicolumn{5}{c|}{ Step $y_{22}$} \\
\hline Method & Rise time & Settl. time & $\%$ Os & $J$ & Rise time & Settl. time & $\%$ Os & $J$ \\
\hline NMS & 5.0 & 28 & 25.0 & 11.5 & 8.0 & 38 & $\mathbf{2 . 7}$ & 12.5 \\
\hline PSO & 4.5 & 28 & 40.0 & 11.2 & 8.0 & $\mathbf{2 2}$ & 35.0 & 11.7 \\
\hline TPO & $\mathbf{4 . 0}$ & $\mathbf{2 0}$ & $\mathbf{2 0 . 0}$ & $\mathbf{7 . 0}$ & 8.0 & $\mathbf{2 2}$ & 6.5 & $\mathbf{1 0 . 3}$ \\
\hline
\end{tabular}

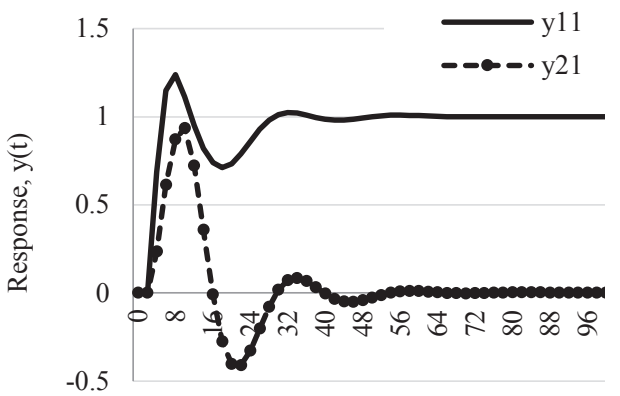

Time (sec)

(a)

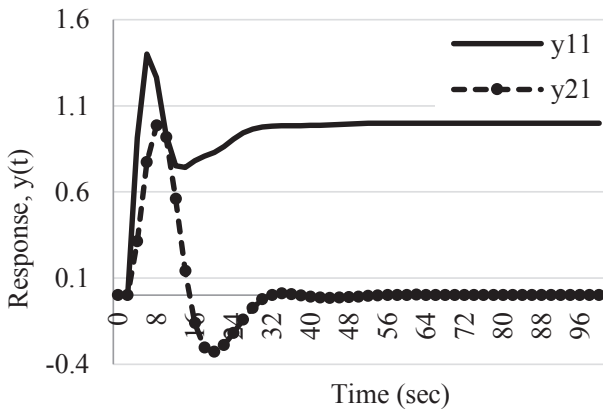

(c)

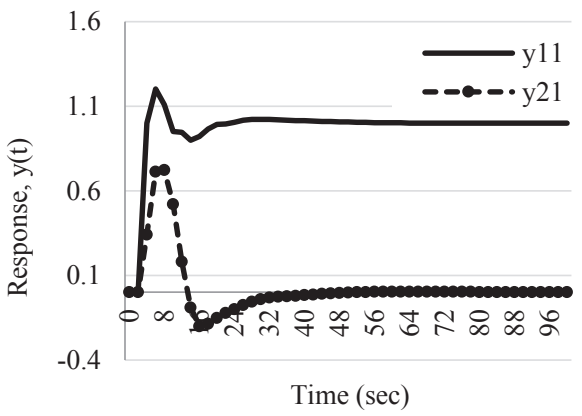

(e)

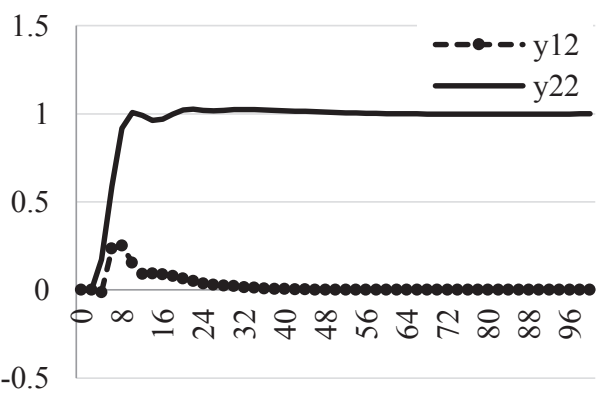

(b)

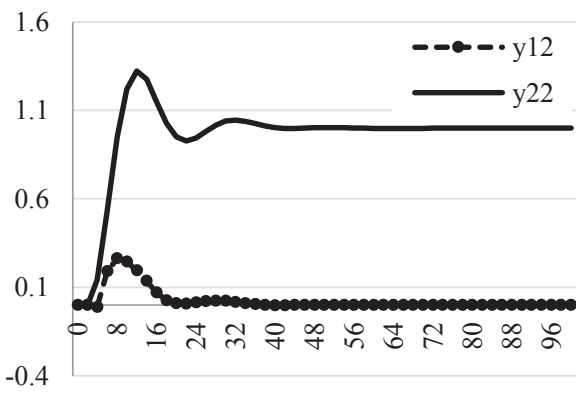

(d)

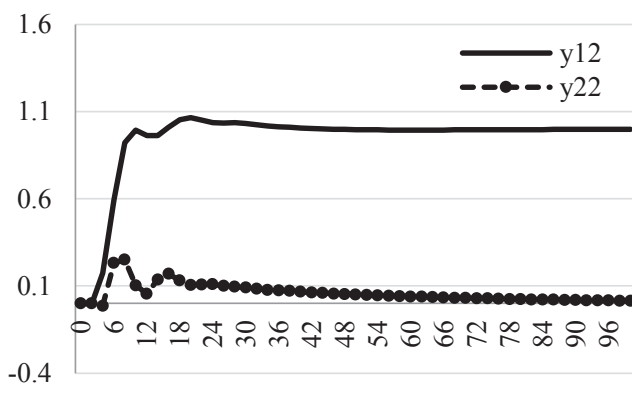

(f)

Fig. 5: Unit step response using NMS for (a) $y 11$ and $y 21$, (b) $y 12$ and $y 22$, PSO for (c) $y 11$ and $y 21$, (d) $y 12$ and $y 22$ TPO for (e) $y 11$ and $y 21$, (f) $y 12$ and $y 22$ 
Based on the response properties, PSO method has the highest overshoot. The performance index as in the table shows that TPO outperformed other algorithms in both steps $y_{11}$ and $y_{22}$ and followed by PSO

\section{Conclusion}

In this paper, the performance of step response for single and multiple variable PID controls for Nelder-Mead simplex, PSO and TPO are compared. For SISO model, TPO outperformed other algorithm in convergence with lesser than $\pm 1 \%$ tolerance. The performance of the algorithm on MIMO control optimization is verified with Wood and Berry distillation column control. TPO shows the best performance index with lower IAE and integral response boundaries. It is also observed that NMS has lower overshoot in step response of $y_{22}$.. The optimum solution of TPO can be improved further by higher number of branches and shoots leaves, as current settings are only 10 branches search and 20 leaves search (which correspond to $10 \times 20$ searches). The search of each branch and leaves are running in parallel; this is to ensure a better diversification and hence also better intensification towards true solution, thus the computational cost is reduced significantly. NMS algorithm is able to search towards global optimum due to its triangularity search and driven with three simplex steps that compares and adapts with better performance during each step. The search of PSO algorithm is driven by the particle location and speed. However PSO search may end up with premature convergence as the algorithm is not able to search diversely in the search space as observed in SISO response. The findings from this paper suggest a necessity of comparison studies with more practical MIMO process and with increased complexity.

The research was supported by Universiti Teknologi PETRONAS. The author would like to thank the university organisation for the support. The author would also like to thank the reviewers for thoughtful comments that added to the efficacy of this paper.

\section{References}

1. Marcio F. Miranda, K. G. Vamvoudakis, "Online optimal auto-tuning of PID controllers for tracking in a special class of linear systems", American Control Conference (ACC), Boston, pp. 5443-5448 (2016).

2. B. L. G. Costa, J.P.L.S., Almeida, B. A. Angelico, "Application of Optimzation Heuristics in Tuning Decentralized PID Controllers". INDUSCON, pp 1-8 (2012)

3. S. Boyd, M. Hast, K. Johan Åström, " MIMO PID tuning via iterated LMI restriction", Int. J. of Robust and Nonlinear Control, pp 1718-1731 (2015)

4. S. Sommer, A. Kienle., " Auto-Tuning of Multivariable PID Controllers Using Iterative Feedback Tuning”, Automatisierungstechnik 60, pp 20-26, OldenbourgWissenschaftsverlag (2012)

5. K. J Åstrom, T. Hägglund, "PID Controller: Theory, Design, and Tuning”, ISA Research Triangle Park, NC (1995)

6. W. L. Luyben., "Simple method for tuning SISO controllers in multivariables systems", Industrial \& Engineering Chemistry Process Design and Development, 25(3), pp 654-660 (1986)

7. O. Roeva, T. Slavov, " PID Controller Tuning based on Metaheuristic Algorithms for Bioprocess Control”, Biotechnology \& Biotechnological Equipment, 26, 5, pp 32673277 (2014) 
8. X. S. Yang, "Nature-Inspired Metaheuristic Algorithms" $2^{\text {nd }}$ Edition, Luniver Press (2010)

9. J. Mange, S. Pace, A. Dunn, "Optimization of PID Controller Parameters for Automated Ground Vehicle Control on Dynamic Terrain", Int'l Conf. Scientific Computing, CSC'15, pp 3-8 (2015)

10. G. B. Dantzig, "Origins of Simplex Method", Technical Report SOL 87-5, Systems Optimization Laboratory (1987)

11. J. A. Nelder., R. Mead, "A simplex method for function minimization”, Computer Journal, 7, pp 308-313 (1965)

12. J. Kennedy, R. Eberhardt, "Particle swarm optimization", proc. of the IEEE Int. Conf. on Neural Networks, Piscataway, NJ, pp 1942-1948 (1995)

13. Aishwarya Y., S. Wadhwani, A. K. Wadhwani, "Particle Swarm Optimization Based Intelligent Tuning of PID Controller for DC Servo Motor Control", Int. Journal of Scientific Engineering and Technology Research, IJSETR, ISSN 2319-8885, 03, Issue 15, pp 3106-3110 (2014)

14. R. K. Wood, M. W. Berry, "Terminal composition of a binary distillation column", Chemical Engineering Science, 28, pp. 1707-1717 (1973)

15. A. Hanif Halim, I. Ismail, "Nonlinear plant modeling using neuro-fuzzy system with Tree Physiology Optimization", IEEE Student Conference on Research and Development (SCOReD) (2013)

16. A. Hanif Halim, I. Ismail, " Online PID controller tuning using tree physiology optimization”, International Conference on Intelligent and Advanced Systems (ICIAS), pp 1- 5 (2016)

17. A. Hanif Halim, I. Ismail, "Comparative Study of Meta-heuristics Optimization Algorithm using Benchmark Function", International Journal of Electrical and Computer Engineering, IJECE, 7, No 2, (2017) 